\title{
The frailty and mortality relationship in patients with COVID-19
}

\author{
Kenneth Jordan $\mathrm{Ng}$ Cheong Chung ${ }^{1}$ (D)
}

Received: 15 August 2020 / Accepted: 29 August 2020 / Published online: 3 January 2021

(c) European Geriatric Medicine Society 2021

Keywords Respiratory tract diseases · COVID-19 $\cdot$ Frailty $\cdot$ Mortality

Dear Editor,

I read with great interest the brief report by Knopp et al. [1] entitled 'Presenting features of COVID-19 in older people: relationships with frailty, inflammation and mortality.' The authors described the clinical characteristics and outcomes of older adults diagnosed with COVID-19. Results of particular interest include higher Clinical Frailty Scale (CFS) scores with decreases in C-reactive protein (CRP) $(p<0.01)$, an age-related increase in mortality (HR 1.1, 95\% CI $1.0-1.1, p<0.01$ ) but neither sex nor frailty was associated with mortality and a higher likelihood of death with rising CRP or higher neutrophil:lymphocyte ratio. I would like to thank the authors for highlighting the importance of the relationship between frailty, inflammation and mortality, especially during the COVID-19 pandemic. Both frailty and inflammation have been linked to increased mortality, especially in patients with COVID-19 [2]. However, I would like to comment on some of their findings which need more clarity.

First, the authors described recording data on ethnicity. I would like to highlight the fact that studies have found a correlation between the severity of COVID-19 and ethnicity with patients of the black, Asian and minority ethnic (BAME) group being more severely affected by COVID19 [3]. Risk of death is particularly high for Black African (HR 3.24, 95\% CI 2.90-3.62) and Pakistani (HR 3.29, 95\% CI 2.96-3.64) groups after adjustment for age and region [3]. Having collected this data, adjustment or an attempt to include it in their results would have been informative to better interpret their findings and compare with other research.

Kenneth Jordan $\mathrm{Ng}$ Cheong Chung

k.ng-cheong-chung@newcastle.ac.uk

1 Translational and Clinical Research Institute, Faculty of Medical Sciences, The Medical School, Newcastle University, Framlington Place, Newcastle upon Tyne NE2 4HH, UK
Second, the authors suggest that frailty on the CFS is not associated with increased mortality. Previous studies have shown that there exist an association between frailty on the CFS and mortality in older inpatients with COVID-19 [4]. More specifically, the CFS has been shown to be a useful tool for risk stratification in patients with COVID-19 and has been shown to better predict disease outcomes than age and comorbidity [2]. This highlights the importance of treating their findings with caution and encouraging further research on this relationship.

Third, they also show an inverse relationship between CRP and frailty measured on the CFS. Although the relationship between frailty and inflammation is poorly understood, the literature suggests frailty and pre-frailty are associated with higher inflammatory parameters with frail and pre-frail patients having significantly higher serum levels of CRP and IL-6 than robust patients [5]. Perhaps adjustment for factors exhibiting worsening clinical outcome with increasing frailty, including CRP, are required to find the true association between frailty and increased mortality as previously described [2].

Author contributions KJNCC contributed to writing and drafting the article.

Funding None.

Data availability Not applicable.

\section{Compliance with ethical standards}

Conflict of interest The author declare that they have no conflict of interest.

Ethics approval Not applicable. 


\section{References}

1. Knopp P, Miles A, Webb TE, McLoughlin BC, Mannan I, Raja $\mathrm{N}$ et al (2020) Presenting features of COVID-19 in older people: relationships with frailty, inflammation and mortality. Eur Geriatr Med 2020:1-6. https://doi.org/10.1007/s41999-020-00373-4

2. Hewitt J, Carter B, Vilches-Moraga A, Quinn TJ, Braude P, Verduri A et al (2020) The effect of frailty on survival in patients with COVID-19 (COPE): a multicentre, European, observational cohort study. Lancet Public Health. https://doi.org/10.1016/s2468 -2667(20)30146-8

3. Aldridge RW, Lewer D, Katikireddi SV, Mathur R, Pathak N, Burns R et al (2020) Black, Asian and Minority Ethnic groups in England are at increased risk of death from COVID-19: indirect standardisation of NHS mortality data. Wellcome Open Res 5:88. https://doi.org/10.12688/wellcomeopenres.15922.2

4. Aw D, Woodrow L, Ogliari G, Harwood R (2020) Association of frailty with mortality in older inpatients with Covid-19: a cohort study. Age Ageing. https://doi.org/10.1093/ageing/afaa184

5. Soysal P, Stubbs B, Lucato P, Luchini C, Solmi M, Peluso R et al (2016) Inflammation and frailty in the elderly: a systematic review and meta-analysis. Ageing Res Rev 31:1-8. https://doi. org/10.1016/j.arr.2016.08.006

Publisher's Note Springer Nature remains neutral with regard to jurisdictional claims in published maps and institutional affiliations. 